\title{
Efectividad de la hidroterapia para disminuir el dolor y mejorar la calidad de vida y función física en adultos con osteoartritis de rodilla: revisión sistemática
}

\author{
J. L. Ibarra Cornejoํㅜ D. G. Quidequeo Reffers², D. A. Eugenin Vergara², E. A. Beltrán Maldonado², \\ S. R. Ricci Muñoz y M. J. Fernández Lara ${ }^{3}$
}

${ }^{1}$ Kinesiología. Centro Biokine Salud y Fitness. San Vicente de Tagua. Tagua, Chile. ${ }^{2}$ Kinesiología. Escuela de Kinesiología. Facultad de Medicina. Universidad Austral de Chile. Chile. ${ }^{3}$ Enfermería. Centro de Diálisis Urodial. San Vicente de Tagua. Tagua, Chile

Ibarra Cornejo JL, Quidequeo Reffers DG, Eugenin Vergara DA, Beltrán Maldonado EA, Ricci Muñoz SR, Fernández Lara MJ. Efectividad de la hidroterapia para disminuir el dolor y mejorar la calidad de vida y función física en adultos con osteoartritis de rodilla: revisión sistemática. Rev Soc Esp Dolor 2015; 22(4): 168-174.

\begin{abstract}
Objective: To determine the effectiveness of hydrotherapy to reduce pain, improve quality of life and physical function in adults with knee osteoarthritis, by collecting the various scientific studies of high methodological quality on the date published.

Material and methods: A systematic review of five computerized bibliographic databases was performed: PEDro and MEDLINE. We included randomized controlled trials with patients diagnosed with knee osteoarthritis trials. Articles published from January 1, 2004 to the July 31, 2014 in Spanish and English were selected. Study screening: Studies were independently selected, unblinded, by two reviewers. The PEDro scale was used to classify the studies.

Results: Of the 119 studies that could potentially have been included. In determining the criteria for inclusion and exclusion only 6 items were selected for analysis.

Conclusions: There is strong evidence that the use of hydrotherapy is effective in the treatment of osteoarthritis of the knee in adults over 50 years with a minimum follow up of 6 weeks.
\end{abstract}

Key words: Hydrotherapy. Osteoarthritis/knee. Pain knee.

Recibido: 22-09-14.

Aceptado: 01-12-14.

\section{RESUMEN}

Objetivo: determinar la efectividad de la hidroterapia para disminuir el dolor, mejorar la calidad de vida y función física en adultos con osteoartritis de rodilla, mediante la recopilación de los diferentes estudios científicos de alta calidad metodológica publicados a la fecha.

Material y métodos: se realizó una revisión sistemática en las bases de datos de: PEDro y MEDLINE. Fueron incluidos ensayos controlados aleatorios con pacientes diagnosticados de osteoartritis de rodilla. Se seleccionaron artículos publicados entre el 1 de enero de 2004 hasta el 31 de julio de 2014 en lengua española e inglesa. Selección de estudios: se realizó de manera independiente, no cegada, por 2 revisores, y se llevó a cabo una clasificación de los estudios mediante la escala PEDro.

Resultados: se encontraron 119 artículos que potencialmente podrían incluirse a este trabajo. Al determinar los criterios de inclusión y exclusión sólo se seleccionaron 6 artículos para su análisis.

Conclusiones: existe evidencia sólida que indica que el uso de hidroterapia es efectiva para el tratamiento de la osteoartritis de rodilla en adultos sobre 50 años, con un seguimiento mínimo de 6 semanas.

Palabras clave: Hidroterapia. Osteoartritis de rodilla. Dolor en rodilla.

\section{INTRODUCCIÓN}

La osteoartritis (OA) es el resultado de factores mecánicos y biológicos que desestabilizan el acoplamiento normal entre degradación y síntesis de matriz extracelular del cartílago articular y hueso subcondral. Esta enfermedad crónica avanza con lentitud y afecta generalmente a personas sobre los 45 años de edad. El Colegio Americano de Reumatología la define como un grupo heterogéneo de patologías con 
manifestaciones clínicas similares y cambios patológicos y radiológicos comunes (1).

La OA tiende a afectar las articulaciones que cargan peso, como las caderas y las rodillas, siendo esta última una de las mayores causas de dolor y discapacidad en el adulto mayor (2). La padece al menos el $15 \%$ de la población mundial por arriba de los 60 años de edad. Inclusive, se ha determinado que dentro de las enfermedades reumáticas la OA es de 10 a 12 veces más frecuente que la artritis reumatoide (3). La OA de rodilla es la más significativa clínicamente y se incrementa con la edad, ya que el 33\% de los hombres y el 53\% de las mujeres de más de 80 años de edad tienen evidencia radiológica de OA, sin embargo, las manifestaciones clínicas sólo se reportan en el 16\% de las mujeres y el 5\% de los hombres de más de 80 años de edad (4).

Esta enfermedad se caracteriza por una destrucción del cartílago articular y del condrocito, además de presentar cambios óseos, deterioro de tendones y ligamentos con varios grados de inflamación de la sinovial (5). Conforme la OA progresa, el cartílago sufre fracturas perpendiculares a la superficie, disminuye la síntesis de colágeno y proteoglicanos, llegando finalmente a la rigidez articular, deformidad y limitación funcional, las cuales afectan negativamente sobre la calidad de vida (6).

El desarrollo de la OA de rodilla es multifactorial, entre ellos se destaca la edad, la predisposición genética, lesiones previas, estrés crónico (desalineamientos) y la obesidad (7). Tambien se ha visto que puede resultar por variaciones en patrones anormales de la marcha como el aumento del momento adductor de rodilla (mayor carga en el compartimiento medial) y disminución del momento adductor de cadera $(8,9)$.

Dentro de los signos y síntomas se destacan principalmente el dolor, la debilidad muscular, pérdida del balance, rigidez e inestabilidad articular. Se ha asociado el dolor con la debilidad y atrofia del músculo cuádriceps en adultos mayores con OA y a su vez se ha demostrado que la disminución de la fuerza de la extremidad inferior está relacionada a un aumento en la discapacidad en las personas con OA. Esto sugiere que la atrofia muscular es una consecuecia o un factor de la OA (10).

El Colegio Americano de Reumatología recomienda realizar actividad física moderada, ya que ha demostrado ser una opción para cambiar positivamente la pérdida de cartílago en los individuos con osteoartritis de rodilla (8). Sin embargo, una gran cantidad de individuos con OA son sedentarios. Los programas de actividad física orientados al fortalecimiento muscular y al entrenamiento aeróbico han obtenido mejoras significativas en el alivio del dolor, la capacidad física y estatus de salud general, sin embargo, estos programas de ejercicios presentan una baja adherencia por parte de los individuos con OA (11). Además, se puede especular que el soporte de peso en las rodillas al realizar estos ejercicios podría causar efectos adversos en la OA(12).

Una posible alternativa son los ejercicios en agua templada (hidroterapia), ya que la flotabilidad reduce las cargas y el daño en la articulación afectada (13). La hidroterapia ha sido usada en programas de terapia física, especialmente porque la ejecución de ejercicios bajo condiciones normales de gravedad son dificultosos y dolorosos en personas con OA. El calor y la presión del agua favorecen la circulación sanguínea y reducen el edema. En consecuencia, un entorno submarino permite la movilización activa temprana y el fortalecimiento dinámico (12).

De lo anteriormente expuesto nace nuestra pregunta de investigación: ¿es efectiva la hidroterapia para disminuir el dolor, mejorar la calidad de vida y función física en adultos con osteoartritis de rodilla? Por ello, el objetivo de la presente revisión es determinar la efectividad de la hidroterapia para disminuir el dolor, mejorar la calidad de vida y función física en adultos con osteoartritis de rodilla, mediante la recopilación de los diferentes estudios científicos de alta calidad metodológica publicados en la fecha.

\section{MATERIALES Y MÉTODOS}

\section{Estrategia de búsqueda}

Se realizó una búsqueda bibliográfica para identificar todos los estudios que pudieran ayudar a dar respuesta a la pregunta de investigación. Se realizó una búsqueda electrónica en las siguientes bases de datos: PEDro (www.pedro. org.au, acceso 31/07/2014), MEDLINE (www.ncbi.nlm. nih.gov/pubmed, acceso 31/07/2014). Se seleccionaron artículos publicados entre el 1 de enero de 2004 hasta el 31 de julio de 2014.

\section{Tipos de estudios}

Para la realización de la presente revisión sistemática se desarrolló una estrategia de búsqueda en la cual se incluyeron ensayos clínicos aleatorizados (ECA) y ensayos clínicos controlados (ECC) que cumplieran con nuestros criterios de elegibilidad.

\section{Criterios de inclusión}

- Artículos que incluyeran adultos.

- Sin distinción de raza.

- Artículos publicados en español e inglés.

- Artículos publicados entre el 1 de enero de 2004 hasta el 31 de julio de 2014.

- Sólo ensayos clínicos aleatorizados y controlados de 
hidroterapia.

- Estudios que presenten una puntuación $\geq 5$ en la escala de PEDro.

\section{Criterios de exclusión}

- Publicaciones con escasa o nula evidencia científica.

- Limitada relevancia profesional. Implicación exclusiva de otras disciplinas.

- Artículos de opinión, monografías, comentarios bibliográficos.

\section{Términos utilizados}

Los términos de búsqueda de nuestra revisión fueron encontraros en el descriptor MeSH (Thesaurus de PubMed) y fueron los siguientes:

- Hydrotherapy.

- Osteoarthritis, knee.

- Pain knee.

\section{Criterios de elegibilidad de los estudios}

A los 119 artículos encontrados por la búsqueda preliminar se les aplicó un filtro con los límites de búsqueda. Luego se realizó una lectura crítica a los títulos y resúmenes, donde fueron incluidos ensayos controlados aleatorizados (ECA) con una puntuación igual o superior a 5/10 en la escala de PEDro (Fig. 1).

\section{Selección de estudios}

Los estudios fueron identificados mediante la estrategia de búsqueda. La evaluación de la elegibilidad de los estudios fue desarrollada de manera independiente, estandarizada y no cegada por 2 revisores, y se llevó a cabo mediante una clasificación de los estudios mediante la escala PEDro (5/10), además de una revisión crítica de cada resumen presentado en los estudios encontrados. En caso de existir desacuerdo entre los revisores este se resolvió mediante consenso y consulta a un tercer revisor.

\section{Análisis de eficacia terapéutica}

Cuando los ECA son heterogéneos no hay un método cuantitativo que permita evaluar el beneficio relativo de la intervención a estudiar contra otras, ya que se estudian diferentes poblaciones o intervenciones. Los estudios analizados en esta revisión incluyeron sujetos de diferentes

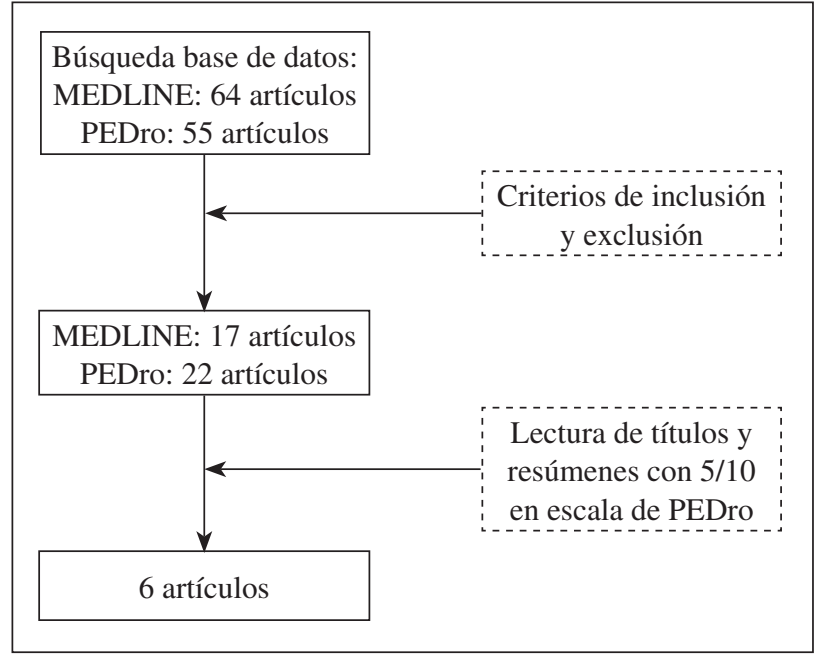

Fig. 1. Algoritmo de búsqueda.

edades, distintos grupos de intervención, diferentes formas de evaluar el tratamiento y diferentes resultados estadísticos, entre otros, por lo que se hace difícil interpretar los resultados de los ensayos individuales. Por este motivo, se utilizará un método cualitativo recomendado por el Grupo Cochrane de Espalda (Van Tulder, 2003) con el uso de niveles de evidencia para la síntesis de los datos (14).

- Evidencia sólida: proporcionada por hallazgos generalmente consistentes en múltiples ECA (3 o más) de alta calidad metodológica.

- Evidencia moderada: proporcionada por resultados generalmente consistentes en 1 ECA de alta calidad metodológica, también se puede interpretar como hallazgos generalmente consistentes en múltiples ECA con moderada calidad metodológica.

- Evidencia limitada: proporcionada por hallazgos consistentes en 1 o más ECA de baja calidad metodológica.

- Evidencia contradictoria: hallazgos no coherentes o inconsistentes en múltiples ECA.

- Ninguna evidencia: ningún ECA encontrado.

\section{Evaluación del riesgo de sesgo en los estudios incluidos}

La escala PEDro (www.pedro.fhs.usyd.edu.au) es un recurso muy utilizado en las investigaciones y los ensayos clínicos de intervenciones fisioterapéuticas. Fue empleada para evaluar el riesgo de sesgo en los estudios incluidos, proporcionando una medida global de la calidad metodológica a través de la valoración de 11 criterios. El criterio n ${ }^{\circ} 1$ evalúa la validez externa y no está incluido en el resultado final. Del criterio 2 al 11 se evalúa la validez interna del artículo con un sistema de puntuación estandarizado (ran- 
go de 0 a 10). Según Moseley (15), los estudios con una puntuación igual o mayor a 5 son calificados como de alta calidad metodológica. El análisis de los estudios incluidos acorde a la escala PEDro fue realizado de manera independiente y sus resultados se presentan en la tabla I.

\section{RESULTADOS}

En la búsqueda se encontraron 119 artículos potencialmente elegibles, los cuales se seleccionaron en función del título y el resumen, y de ellos sólo 6 documentos primarios fueron examinados.

Lund y cols. (16) desarrollaron un ECA para comparar la eficacia de un programa de ejercicios acuáticos y de tierra versus un grupo control para pacientes con OA de rodilla. El estudio contó con 3 grupos, con un periodo de tratamiento de 8 semanas y un periodo de seguimiento de 3 meses. Participaron 79 pacientes con promedios de edad entre 65 y 70 años, con diagnóstico de OA de rodilla que fueron asignados de forma aleatoria en un grupo de ejercicio acuático, un grupo de ejercicio en tierra y un grupo control. El programa de ejercicio acuático se llevó a cabo en una piscina de 1,58 metros de profundidad, en donde se realizó una etapa de calentamiento (correr inmerso de la piscina por 10 minutos), ejercicios de fortalecimiento y resistencia (ejercicios por 20 minutos que incluyen; flexión, extensión y abducción de extremidades inferiores con un peso adicional en los pies), ejercicios de balance, estiramientos y una etapa de enfriamiento, mientras que el programa de ejercicios en tierra se dividió en una etapa de calentamiento en bicicleta estática (10 minutos), ejercicios de fortalecimiento y resistencias (ejercicios por 20 minutos que incluyen: press banca de piernas, pararse y sentarse en una silla, y subir y bajar escaleras), ejercicio de balance, estiramientos y una etapa de enfriamiento, el grupo control no tuvo intervención. Es importante destacar que los ejercicios no fueron individualizados. Se evaluaron la fuerza muscular isométrica de rodilla, dolor con la escala EVA, calidad de vida (cuestionario KOOS), balance y reacciones adversas al ejercicio. En los resultados obtenidos por los autores, no encontraron efectos en ninguna de sus mediciones posterior al término de los ejercicios ( 8 semanas), pero a los 3 meses de seguimiento encontraron una reducción del dolor y aumento de la fuerza muscular sólo en el grupo de ejercicios en tierra comparado con el grupo control, pero no hay mejoras en el seguimiento del grupo de ejercicio acuático; sin embargo, 11 pacientes reportaron eventos adversos en el grupo de ejercicios en tierra, 8 pacientes (32\%) reportaron incremento del dolor durante y después del ejercicio y 3 pacientes $(12 \%)$ reportaron edemas en la rodilla, mientras, sólo 3 pacientes $(11 \%)$ reportaron efectos adversos en el grupo acuático (dolor durante el ejercicio). Los autores concluyen que sólo los ejercicios en tierra mostraron una disminución del dolor y aumento en la fuerza muscular comparado con el grupo control, mientras que no hay beneficios del ejercicio acuático comparado con el grupo control, sin embargo, el ejercicio acuático tiene menos efectos adversos comparado con el grupo de ejercicio en tierra.

Schencking y cols. (17) desarrollaron un estudio piloto, aleatorio y comparativo con el objetivo de determinar los efectos de la hidroterapia en comparación a la fisioterapia convencional en sujetos con OA de rodilla y cadera, para lo cual, tomaron una muestra de 30 pacientes que fueron asignados de manera aleatoria en 3 grupos de 10 participantes cada uno. El tratamiento del grupo 1 consistía en hidroterapia 1 vez al día, el tratamiento del grupo 2 consistía en fisioterapia convencional (estiramientos, fortalecimiento y resistencia específicas de la articulación de la rodilla y cadera, 3 veces a la semana), y el tratamiento del grupo 3 consistía en hidroterapia (diario) con fisioterapia convencional (3 veces a las semana). El periodo de tratamiento fue de 3 meses y fueron reevaluados 10 semanas después de finalizar dicho tiempo de intervención. Se evaluaron el dolor con la escala EVA, calidad de vida y función física (cuestionario SF-36) y movilidad de la articulación (TUG, ROM). En los resultados obtenidos por los autores, encontraron que el grupo 1 tuvo efectos beneficiosos en la movilidad articular y en la disminución del dolor (promedio EVA de base 5,3 a promedio EVA final 2,9) seguido por el grupo 3 (de 4,1 a 2,6). Mientras que se encontró una mejora

TABLA I. RESULTADOS DE LA ESCALA DE PEDro APLICADA A LOS 6 ARTÍCULOS SELECCIONADOS

\begin{tabular}{llllllllllllll}
\hline \multicolumn{1}{c}{ Estudio } & I.1 & I.2 & I.3 & I.4 & I.5 & I.6 & I.7 & I.8 & I.9 & I.10 & I.11 & Total \\
\hline Lund, 2008 & Sí & Sí & Sí & Sí & No & No & Sí & Sí & Sí & Sí & Sí & $8 / 10$ \\
Fransen, 2007 & Sí & Sí & Sí & Sí & No & No & Sí & Sí & Sí & Sí & Sí & $8 / 10$ \\
Hinman, 2007 & Sí & Sí & Sí & Sí & No & No & Sí & Sí & Sí & Sí & Sí & $8 / 10$ \\
Silva, 2007 & Sí & Sí & Sí & Sí & No & No & Sí & Sí & No & Sí & Sí & $8 / 10$ \\
Davey, 2005 & Sí & Sí & Sí & Sí & No & No & Sí & No & Sí & Sí & Sí & $7 / 10$ \\
Schencking, 2013 & Sí & Sí & Sí & Sí & No & No & No & Sí & No & No & Sí & $5 / 10$ \\
\hline
\end{tabular}


en la calidad de vida y función física (SF-36) en el grupo 3 seguido por el grupo 2. Estos efectos se mantuvieron a las 10 semanas de seguimiento. Los autores concluyen que la hidroterapia es un tratamiento eficaz para los pacientes con OA.

Hinman y cols. (18) realizaron un ensayo clínico aleatorizado con el fin de probar la eficacia de un programa de hidroterapia en pacientes con OA de rodilla y/o cadera. El estudio postulaba que el ejercicio físico en un medio acuático presentaría mejoras en el dolor y la función física. También se midieron variables secundarias como rigidez, calidad de vida, actividad física y fuerza muscular. Fueron 71 pacientes reclutados (mayores de 50 años entre hombres y mujeres), los cuales fueron distribuidos en dos grupos de manera aleatoria, quedando 36 en el grupo de actividad física acuática (grupo de intervención) y 35 en el grupo control. Se realizó una evaluación previa (dolor con EVA, función física y rigidez con WOMAC y calidad de vida con el cuestionario de calidad de vida), a todos los pacientes, y luego inmediatamente después de haber terminado las 6 semanas de entrenamiento, sólo al grupo de intervención se le realizó un seguimiento por lo que se reevaluó 6 semanas después de haber terminado el programa. El programa consistía en ejercicios de cargas funcionales y progresivas en una piscina, realizados 2 veces por semana con una duración de 45-60 minutos. Se entrenaban como máximo a 6 pacientes donde el fisioterapeuta experimentado instruyó individualmente a cada paciente palpando la contracción de la musculatura. El grupo control no tuvo entrenamiento pero se le ofreció terapia después de haber terminado el estudio. Los resultados sugieren que 26 pacientes (72\%) del grupo de entrenamiento mejoraron significativamente el dolor global, lo cual corresponde a un 33\% de mejora con respecto al valor inicial, mientras que 6 pacientes (17\%) en el grupo control mostraron resultados positivos. Del mismo modo 27 pacientes $(75 \%)$ del grupo de intervención obtuvieron una mejora en la función física y sólo un 17\% lo hizo en el grupo de control. Los autores concluyen que un programa de seis semanas de hidroterapia produce leves mejoras en dolor, rigidez, fuerza de cadera y calidad de vida, en pacientes con OA de rodilla o cadera, por lo que la hidroterapia se considera una intervención útil para este tipo de pacientes.

Fransen y cols. (19) determinaron los beneficios clínicos del Tai Chí y clases de hidroterapia en pacientes con osteoartritis de cadera o rodilla. Para ello reclutaron a un total de 152 pacientes de entre 59 a 85 años de edad con OA de rodilla o cadera, con sintomatología por más de un año, con capacidad de marcha sin asistencia fuera del hogar. Excluyeron a pacientes que realizaran actividad física recreacional 2 o más veces a la semana, con diagnósticos de: enfermedades cardiacas inestables o respiratorias severas; síndromes de incontinencia; temor al agua; epilepsia descontrolada; dolor lumbar referido a miembros inferio- res o pacientes sometidos a artroscopias o con reemplazo articular por prótesis. Los participantes fueron repartidos aleatoriamente en 3 grupos: 33 pacientes en grupo hidroterapia, 56 en grupo Tai Chí y 41 en grupo control. Los grupos de intervención realizaron clases de una hora dos veces por semana durante 12 semanas con una duración de 60 minutos, sumado a la opción de practicar ejercicios en el hogar no monitorizados. Las clases de hidroterapia fueron guiadas por fisioterapeutas, basadas en un protocolo de ejercicios progresivos en repeticiones y velocidad con periodos de pausas de hidratación, llevados a cabo con el nivel de agua a la altura de la cintura, con temperatura del agua a $34{ }^{\circ} \mathrm{C}$. Las clases de Tai Chí fueron guiadas por instructores del área, basados en el "Tai Chi for Athritis video, Paul Lam". Se realizaron mediciones pre-intervención, post-intervención y seguimiento a las 12 semanas de: dolor y función física (WOMAC), estado de bienestar psicológico (DASS21), rendimiento físico (up an go test, timed 50-foot walk test y stair climb test), efectividad del tratamiento por parte del paciente por los síntomas articulares (SF-12) y recurrencia de sintomatología articular (SF12). Los resultados evidencian mejoras post-tratamiento en el dolor y función para ambos grupos de intervención. El grupo de hidroterapia mostró mejoras significativas en todas las mediciones de rendimiento físico y estado de salud global sobre el grupo control, el grupo Tai Chí sólo evidenció mejoras en la prueba stair climb de rendimiento físico, comparado a su mismo grupo. Además, los autores destacaron que las clases de hidroterapia parecían ser más aceptables y llevaderas (mayor asistencia).

Davey y cols. (20) determinaron la efectividad de la hidroterapia para el manejo de la osteoartritis de miembro inferior en pacientes ancianos; participaron 312 pacientes (196 mujeres y 116 hombres), con edades sobre 60 años que tuviesen OA confirmada en cadera y/o rodilla. Los pacientes fueron asignados en 2 grupos de manera aleatoria: 153 participantes en un grupo de hidroterapia y 159 en un grupo control. El grupo de hidroterapia realizó una etapa de calentamiento, ejercicios de fortalecimiento, de rango articular, coordinación y balance en una piscina de 1,40 metros 2 veces por semana con una duración de 60 minutos. Hubo progresión de cargas con flotadores cada 6-8 semanas. El grupo control recibió el cuidado convencional: sólo se realizaron entrevistas telefónicas semi-estructuradas cada 3 meses. La intervención principal se extendió por 1 año, con un seguimiento por 6 meses. Se evaluaron el dolor, rigidez y función física con el indice de WOMAC, calidad de vida con SF-36 y costo-efectividad EuroQol (EQ-VAS y EQ 5D). Los resultados evidencian mejoras significativas entre los grupos de entrenamiento acuático y control para dolor $(0,026)$, rigidez $(0,0191)$ y función física $(0,0003)$ a las 12 semanas de entrenamiento. Con respecto a la calidad de vida (SF-36) se observaron resultados significativos entre el grupo control y de entre- 
namiento a los 6 y 12 meses de entrenamiento $(0,001)$ y 6 meses de seguimiento $(0,012)$. Después de 6 meses terminado el tratamiento, se mantienen las diferencias significativas en el índice WOMAC para dolor, rigidez y función física $(0,007,0,019$ y 0,013 respectivamente). Los autores concluyen que la hidroterapia por más de 1 año puede producir una reducción significativa en el dolor y mejoría de la función física en los adultos mayores con OA de miembro inferior y puede ser un complemento útil al tratamiento de OA de cadera/rodilla.

De igual manera, Silva y cols. (21) destacan que la hidroterapia proporciona una disminución en el dolor (EVA) y una mejora en la función física (WOMAC) en pacientes con OA de rodilla en comparación con el grupo de ejercicios en tierra después de 3 sesiones a la semana durante 18 semanas de intervención con una duración de 50 minutos. Los autores concluyen que la hidroterapia es una alternativa adecuada y eficaz para disminuir el dolor y mejorar la calidad de vida en el tratamiento de la OA de rodilla.

\section{DISCUSIÓN}

Se intentó determinar si la hidroterapia presenta mejores resultados clínicos que el ejercicio en tierra en el manejo del dolor, función física y calidad de vida. Para lograrlo se realizó una síntesis de la evidencia disponible y de alta calidad metodológica para responder al interrogante planteado precedentemente. Una vez realizada la búsqueda sistemática y la categorización de los estudios con los criterios de inclusión y exclusión, se obtuvieron 6 artículos con buena calidad metodológica y bajo riesgo de sesgo, obteniéndose así una evidencia sólida que indica que la hidroterapia es efectiva para reducir el dolor, mejorar la calidad de vida y función física en pacientes con OA de rodilla, ya que 5 de los 6 artículos presentaron una disminución del dolor a los 3 meses de tratamiento y un aumento sustancial en la calidad de vida y función física en comparación al grupo control (17-21). Sin embargo, estos resultados son para pacientes con rangos de edad entre 52 a 86 años, con un entrenamiento mínimo de 2 veces a la semana y una duración de 60 minutos cada una (17-21). En los 5 artículos que encontraron resultados a favor de la hidroterapia describieron un entrenamiento de cargas progresivas de extremidades inferiores, mientras que el artículo que no evidenció disminución del dolor y aumento de la calidad de vida con la hidroterapia, no presentó un entrenamiento individualizado, sino más bien un protocolo de ejercicios poco específicos para cada paciente (16).

De los 6 artículos, dos destacaron que la hidroterapia presenta mayor adherencia al tratamiento por parte de los pacientes, indicando mayor asistencia a las sesiones $(16,19)$. Lund planteó que esta adherencia al tratamiento se debe a la menor incidencia de reaciones adversas ocurridas durante y posterior a la hidroterapia versus el ejercicio en tierra (16). En todos los estudios se hizo un seguimiento mínimo hasta la sexta semana, por lo que la hidroterapia presenta una evidencia sólida a corto plazo, es decir, hasta la sexta semana.

Resultados similares obtuvieron Bartels y cols. (22) en su revisión sistemática publicada en Cochrane en 2009. Esta revisión incluyó todos los artículos, comentarios y entrevistas sobre hidroterapia publicados a la fecha, sin importar su calidad metodológica, en donde concluyeron que los ejercicios acuáticos parecen tener ciertos beneficios a corto plazo ( 3 meses) para pacientes con artrosis de cadera y/o rodilla. Comparado con no hacer ejercicio o con ejercicios en suelo, los ejercicios en el agua reducen el dolor y mejoran la funcionalidad. Por otro lado, Hall y cols. (23), en un metaanálisis que evaluó el ejercicio acuático para mejorar el dolor en adultos con alteraciones neurológicas u osteoarticulares, realizaron una búsqueda de todos los artículos existentes desde 1980 hasta el año 2006 pero, al igual que en el artículo anterior, no se consideró la calidad metodológica de los estudios incluidos y se concluyó que no hay diferencias entre ejercicios en tierra y acuáticos para mejorar el dolor, pero sí hay diferencias con el grupo control.

\section{CONCLUSIÓN}

Independientemente de la homogeneidad clínica que presentaban todos los artículos seleccionados por nuestra revisión, la alta heterogeneidad estadística no permitió el agrupamiento estadístico de los datos basados en un estimador puntual. Aun así, igual se consideró pertinente realizar un agrupamiento cualitativo o descriptivo de los resultados en las intervenciones clínicamente similares.

Según los resultados arrojados por los estudios primarios incluidos en nuestra revisión, existe evidencia sólida que la hidroterapia es efectiva en la disminución del dolor y mejora de la calidad de vida y función física en pacientes con osteoartritis de rodilla con 6 semanas de seguimiento.

Cabe consignar que las conclusiones aportadas por nuestra revisión fueron realizadas en función de los artículos localizados por nuestra estrategia de búsqueda y seleccionados bajo nuestros criterios de elegibilidad, por lo tanto, siempre existe la probabilidad de que haya estudios que por problemas de indexación o límites de búsqueda no hayan sido incorporados en esta revisión.

\footnotetext{
CORRESPONDENCIA:

José Luis Ibarra Cornejo

Kinesiología

Centro Biokine Salud y Fitness

San Vicente de Tagua

Tagua, Chile

e-mail: jose.ibarra.cornejo@gmail.com
} 


\section{BIBLIOGRAFÍA}

1. Altman R, Asch E, Bloch D, Bole G, Borenstein D, Brandt $\mathrm{K}$, et al. The American College of Rheumatology criteria for the classification and reporting of osteoarthritis of the knee. Arthritis Rheum 1986;29:1039-49.

2. Cooper C, Snow S, Mcalindon T, kellingray S, Stuart B, Coggon D, et al. Risk factors for the incidence and progression of radiographic knee osteoarthritis. Arthritis \& Rheumatism 2000;43(5):995-1000.

3. Wieland HA, Michaelis M, Kirschbaum BJ, Rudolphi KA. 2005. Osteoarthritis-an untreatable disease? Nat Rev Drug Discov 2005;4:331-44.

4. Felson DT,Naimark A, Anderson J, Kazis L, Castelli W, Meenan RF. The prevalence of knee osteoarthritis in the elderly. The Framingham Osteoarthritis Study. Arthritis Rheum 1987;30:914-8.

5. Rikulmontree T. Osteoarthritis [Internet] 2012. [updated 2012; cited 2012 Dec 03]. Available at: http://www.rheumatology.org/practice/clinical/patients/diseases_and_conditions/osteoarthritis.asp.

6. Cooper C. Osteoarthritis: Epidemiology and classification. In: Hochberg MC, editor. Rheumatology. 3rd ed. Toronto, Ontario, Canada: Mosby; 2003. p. 1781-91.

7. Kerr RG, Al-Kawan RH. Osteoarthritis: A primary care approach for physicians in 2000 and beyond. Saudi Med J 2001;22:403-6.

8. Woollard J, Gil A, Sparto P, Kwoh K, Piva S, Farrokhi S, et al. Change in knee cartilage volume in individuals completing a therapeutic exercise program for knee osteoarthritis. J Orthop Sports Phys Ther 2011; 41(10):708-22. doi:10.2519/ jospt.2011.3633.

9. Hurwitz DE, Sumner DR, Andriacchi TP, Sugar DA. Dynamic knee loads during gait predict proximal tibial bone distribution. J Biomech 1998;31(5):423-30.

10. Foley A, Halbert J, Hewitt T, Crotty M. Does hydrotherapy improve strength and physical function in patients with osteoarthritis a randomised controlled trial comparing a gym based and a hydrotherapy based strengthening programme. Ann Rheum Dis 2003;62:1162-7. doi: 10.1136/ ard.2002.005272.

11. Munro JF, Nicholl JP, Brazier JE, Davey R, Cochrane T. Cost effectiveness of a community based exercise programme in over 65 year olds: Cluster randomised trial. J Epidemiol Community Health 2004;58:1004-10.

12. Rogind H, Bibow-Nielsen B, Jensen B, Moller HC, Frimodt-Moller H, Bliddal $\mathrm{H}$. The effects of a physical training program on patients with osteoarthritis of the knees. Arch Phys Med Rehabil 1998;79:1421-7.

13. Biscarini A, Cerulli G. Modeling of the knee joint load in rehabilitative knee extension exercises under water. J Biomech 2006;17:1-11.

14. Van Tulder M, Furlan A, Bombardier C, Bouter L. Editorial Board of the Cochrane Collaboration Back Review Group. Updated Method Guidelines for Systematic Reviews in the Cochrane Collaboration Back Review Group. Spine 2003;28(12):1290-9.

15. Moseley AM, Herbert RD, Sherrington C, Maher CG. Evidence for physiotherapy practice: A survey of the Physiotherapy Evidence Database (PEDro). Aust J Physiother 2002;48:43-9.

16. Lund H, Weile U, Christensen R, Rostock B, Downey A, Bartels EM. A randomized controlled trial of aquatic and land-based exercise in patients with knee osteoarthritis. J Rehabil Med 2008;40:137-44.

17. Schencking M, Wilm S, Redaelli M. A comparison of Kneipp hydrotherapy with conventional physiotherapy in the treatment of osteoarthritis: A pilot trial. J Integr Med 2013;11(1):17-25.

18. Hinman R, Heywood S, Day A. Aquatic physical therapy for hip and knee osteoarthritis: Results of a single-blind randomized controlled trial. Physical Therapy 2007;87(1).

19. Fransen M, Nairn L, Winstainley J, Lam P, Edmonds J. Physical activity for osteoarthritis management: A randomized controlled clinical trial evaluating hydrotherapy or Tai Chi classes. Arthrutus \& Rheumatism 2007;57(3):407-14.

20. Davey R, Matthes S. Randomised controlled trial of the cost-effectiveness of water-based therapy for lower limb osteoarthritis. Health Technology Assessment 2005;9(31).

21. Silva L, Valim V, Pessanha A, Oliveira L, Myamoto S, Jones A, et al. Hydrotherapy versus conventional land-based exercise for the management of patients with osteoarthritis of the knee: A randomized clinical trial. Phys Ther 2008;88:12.

22. Bartels EM, Lund H, Hagen KB, Dagfinrud H, Christensen R, Danneskiold- Samsøe B. Aquatic exercise for the treatment of knee and hip osteoarthritis. Cochrane Reviews. 2007, Issue 4. Art No: CD005523. DOI:10.1002/14651858. CD005523.pub2.

23. Hall J, Swinkels A, Briddon J, McCabe CS. Does aquatic exercise relieve pain in adults with neurologic or musculoskeletal disease? A systematic review and meta-analysis of randomized controlled trials. Arch Phys Med Rehabil 2008;89:873-83. 